\section{$\underset{\substack{\text { hommes } \\ \text { \& migrations }}}{ }$}

\section{Hommes \& migrations}

Revue française de référence sur les dynamiques

migratoires

$1284 \mid 2010$

Migrations et environnement

\title{
Le projet EACH-FOR
}

\section{Han Entzinger, Jill Jäger et François Gemenne}

\section{(2) OpenEdition \\ Journals}

\section{Édition électronique}

URL : http://journals.openedition.org/hommesmigrations/1232

DOI : 10.4000/hommesmigrations. 1232

ISSN : 2262-3353

\section{Éditeur}

Musée national de l'histoire de l'immigration

\section{Édition imprimée}

Date de publication : 1 mars 2010

Pagination : 10-15

ISSN : 1142-852X

Référence électronique

Han Entzinger, Jill Jäger et François Gemenne, «Le projet EACH-FOR », Hommes \& migrations [En ligne], 1284 | 2010, mis en ligne le 29 mai 2013, consulté le 24 avril 2019. URL : http://

journals.openedition.org/hommesmigrations/1232; DOI : 10.4000/hommesmigrations.1232 


\section{Le projet EACH-FOR}

Par Han Entzinger, Université de Rotterdam

Jill Jäger, Sustainable Europe Research Institute, Vienne et François Gemenne, chercheur en sciences politiques, CEDEM - ULg / Iddri - Sciences Po

La plupart des études de cas présentées dans ce dossier ont été réalisées dans le cadre du projet EACH-FOR (Environmental Change and Forced Migration Scenarios, www.each-for.eu). Ce projet a été financé par la Commission européenne entre 2007 et 2009 et a été réalisé par sept instituts de recherche européens. Le projet visait à documenter, de manière empirique, la façon dont les dégradations de l'environnement pouvaient influer sur les comportements migratoires dans différents contextes. Deux objectifs spécifiques avaient été fixés : d'une part, explorer et décrire les causes de migration liées aux changements de l'environnement ; d'autre part, élaborer des scénarios plausibles d'évolution des migrations induites par les changements environnementaux.

La question centrale qui a motivé le projet touchait au rôle des dégradations environnementales dans la décision migratoire. Il existait certes de nombreuses recherches théoriques sur le sujet, mais ces recherches ne s'appuyaient pas sur un nombre suffisant d'études empiriques, comparables entre elles. L'équipe de recherche a donc développé une méthodologie spécifique pour analyser le rôle de l'environnement dans la décision migratoire, et a testé cette méthodologie dans un grand nombre d'études de cas, de manière à pouvoir tirer certaines observations empiriques quant à la relation entre environnement et migrations dans différents contextes. Dès le départ, le projet était conçu comme un projet exploratoire : il s'agissait avant tout d'examiner quelle méthodologie pouvait être utilisée, et de tirer quelques observations préliminaires. Ce projet, idéalement, devrait se poursuivre dans une deuxième phase, qui permettrait d'étudier certains cas de manière plus approfondie.

\section{Études de cas et méthodologie}

Un élément essentiel du projet fut donc la réalisation de 23 études de cas à travers le monde, de manière à offrir une grande diversité de contextes, et notamment une grande variété de dégradations environnementales. Leur choix fait suite à la 
réalisation d'études exploratoires à l'échelle régionale qui ont permis d'identifier les situations les plus pertinentes pour le projet. Parmi ces 23 études de cas, représentées sur la carte ci-dessous, six sont présentées dans ce dossier : il s'agit de celles réalisées au Bangladesh, en Mongolie intérieure (Chine), dans l'île d'Hispaniola (Haïti et République dominicaine), au Kirghizistan, au Maroc et au Mozambique.

Dans certains pays, les dégradations de l'environnement étaient directement liées au changement climatique - par exemple le cas de l'élévation du niveau des mers au Bangladesh -, mais pas du tout dans d'autres cas - par exemple le stockage de déchets nucléaires au Kirghizistan. Certaines dégradations étaient subites, comme les inondations du fleuve Zambèze au Mozambique, d'autres, plus progressives, comme la désertification au Maroc. Les études de cas présentées dans ce dossier reflètent cette variété de dégradations de l'environnement, afin de fournir un aperçu le plus large possible de la manière dont différentes modifications de l'environnement affectent les comportements migratoires.

Toutes les études de cas du projet, à l'exception de celles menées en Europe, qui ne sont pas présentées dans ce dossier, ont été réalisées à partir d'études de terrain. Ces études de terrain comportaient la réalisation d'entretiens semi-directifs avec des experts locaux (autorités, ONG, chercheurs, etc.) ainsi qu'un questionnaire uniformisé, à partir duquel étaient interrogés à la fois ceux qui avaient migré et ceux 
qui étaient restés dans la région dont l'environnement était dégradé (les "nonmigrants"). Le travail de terrain cherchait à découvrir : qui étaient ceux qui avaient quitté les régions frappées par des dommages environnementaux; d'où provenaient les migrants, et où ils se dirigeaient ; pourquoi les migrants avaient migré ; comment les facteurs environnementaux se mêlaient à des facteurs économiques, sociaux et politiques dans la décision migratoire; les obstacles qui empêchaient parfois la migration; les raisons pour lesquelles certaines personnes demeuraient dans les régions touchées par des dégradations de l'environnement, tandis que d'autres migraient ; comment s'organisaient les migrations ; et enfin quels étaient les effets de ces migrations sur la région d'origine.

Dans un premier temps, des experts locaux étaient interrogés, dans des entretiens semi-directifs. Les experts étaient à la fois des représentants des autorités et des organisations internationales, mais aussi des membres d'organisations non gouvernementales et des chercheurs. Ces entretiens ont permis l'identification des endroits où les questionnaires aux migrants pouvaient être administrés, ainsi qu'une compréhension plus profonde des processus environnementaux et migratoires à l'ceuvre dans la région. 
Dans un deuxième temps, le travail de terrain consistait à interroger des migrants et des "non-migrants", à l'aide d'un questionnaire standardisé. Le questionnaire couvrait l'ensemble des questions décrites ci-dessus, en cherchant à analyser le parcours des migrants et ses déterminants. En raison de limitations de temps et de budget, seuls 30 migrants et 30 non-migrants environ ont pu être interrogés dans chaque étude de cas. L'analyse des questionnaires ne permet donc pas un traitement statistique en vue d'une analyse quantitative, mais elle livre néanmoins de nombreux enseignements.

\section{Principaux enseignements}

Bien qu'il ait une vocation essentiellement exploratoire, le projet EACH-FOR reste un projet important, car il constitue le tout premier projet empirique à effectuer une comparaison systématique entre des études de cas très variées, réalisées selon une méthodologie commune. À ce titre, il renseigne sur la réalité empirique des migrations environnementales, souvent bien différente des discours dominants sur la question. En premier lieu, le projet a mis en évidence que les dégradations de l'environnement, dans les régions étudiées, n'étaient pas les seuls déterminants du comportement migratoire. D'autres facteurs, d'ordre économique, social ou politique, se mêlent à la décision migratoire, et il est souvent impossible de les démêler les uns des autres. Néanmoins, la magnitude et la fréquence des risques environnementaux se multiplient, notamment sous l'impact du changement climatique, accroissant de ce fait la pression à la migration.

La migration n'est pas toujours le résultat d'une incapacité à s'adapter aux changements de l'environnement. Dans de nombreux cas, des processus traditionnels de migration se sont développés précisément comme un moyen de faire face à des bouleversements habituels de l'environnement. Ces processus de migration, néanmoins, connaissent des transformations profondes à la suite des dégradations de l'environnement et/ou des conditions socio-économiques. La migration, en général, se produit lorsqu'il devient impossible de tirer des revenus suffisants de l'activité habituelle. C'est le cas en particulier lorsqu'une activité agricole ou d'élevage est affectée par une dégradation progressive de l'environnement ou des événements extrêmes. Nos recherches ont clairement indiqué que ceux qui sont propriétaires de leurs terres agricoles ont une plus grande tendance à y rester le plus longtemps possible que ceux qui sont employés. Il est évident que le départ des agriculteurs a un impact considérable sur toute autre activité économique dans la région et déclenche souvent un vaste exode rural. 
Contrairement à une idée souvent véhiculée, les migrations liées à l'environnement ne sont pas toujours temporaires: les migrations de long terme ou permanentes deviennent de plus en plus fréquentes, en particulier parmi les jeunes générations. La migration, néanmoins, reste un processus coûteux : ceux qui peuvent partir ne peuvent le faire que s'ils disposent des ressources suffisantes, ainsi que d'un accès aux réseaux qui facilitent la migration. Dans de nombreux cas, les populations vulnérables se trouveront donc incapables de migrer, et directement exposées aux dégradations de leur environnement. Si ces dernières débouchent sur une impossibilité physique de rester, comme dans le cas des inondations, les victimes de tels événements ont tendance à s'installer à proximité immédiate de leur lieu d'origine et d'y retourner dès que possible. Pourtant, très souvent, une certaine sélection apparait : les jeunes partent ailleurs et les vieux retournent dans leur région d'origine. Il est évident que cette situation nuit beaucoup au rétablissement ultérieur de la région affectée. 
Pour la Commission européenne, un motif important de financement du projet EACH-FOR était l'idée que les dégradations de l'environnement à travers le monde pourraient intensifier les flux migratoires vers l'Europe. Nos recherches n'ont pas confirmé cette hypothèse. Ce n'est que dans les cas où il y a une tradition migratoire préexistante vers l'Europe que certaines des victimes de la dégradation environnementale ont tendance à rejoindre leurs proches qui ont déjà émigré en

Europe. C'est, par exemple,

le cas pour le Maroc et pour la République dominicaine, ainsi que pour l'Égypte (ce cas faisait également partie du projet, mais n'est pas repris dans ce dossier spécial). Quelles seraient les solutions au niveau politique pour traiter la question des migrations liées à la dégradation de l'environnement? Le projet EACH-FOR a suggéré deux pistes. La première est d'étudier s'il est nécessaire de créer un statut international spécifique pour les personnes qui se voient forcées de quitter leur pays pour des raisons environnementales. Un tel statut a été proposé par certains, qui le comparent à celui des réfugiés politiques. Pourtant, d'autres sont de l'avis que, bien que le déplacement puisse être forcé, il ne s'agit pas de réfugiés au sens propre du terme, et qu'il ne faudrait pas "diluer" les instruments de protection qui existent dans ce domaine. La deuxième piste est de nature plus structurelle et vise à une politique de développement soutenable qui réduit autant que possible les effets nuisibles du changement climatique, y compris les départs forcés. Ceci demande, entre autres, une plus étroite liaison entre les politiques de développement et celles des migrations, tant au niveau bilatéral qu'au niveau multilatéral. 